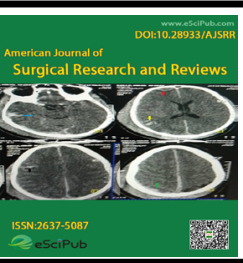

American Journal of Surgical Research and Reviews

(ISSN:2637-5087)

\title{
A comparative study between laparoscopic hernia repair and open herniotomy of inguinal hernia in paediatric age group: A prospective randomized controlled study
}

Dr.Arti Mitra1, Dr.Unmed Chandak ${ }^{1}$, Dr.Yuvraj Pawaskar ${ }^{1 *}$, Dr.Shiv Kumar Sahu ${ }^{1}$, Dr. Sanskriti Sinha', Dr. Prasad Bansod", Dr. Nilesh Nagdeve², Dr. Akanksha Waldia ${ }^{3}$

${ }^{1}$ Department of General surgery, Government Medical College and Hospital Nagpur, Maharashtra, India-440003; 'Department of Paediatric surgery, Government Medical College and Hospital Nagpur, Maharashtra, India-440003; ${ }^{3}$ MBBS Intern, Indira Gandhi Government Medical College \& Hospital, Nagpur, Maharashtra, India-440012

\section{ABSTRACT}

Background: Inguinal hernia in children remains one of the most *Correspondence to Author: common congenital anomaly observed by surgeons. Prompt diagnosis and early treatment of the inguinal hernia continues to be the mainstay to avoid the complications. The present study was undertaken to compare the effectiveness of laparoscopic hernia repair and open herniotomy of inguinal hernia in paediatric age group. Method: A total 104 healthy children of age $<12$ years diagnosed with inguinal hernias were alternately randomized into two equal groups, 52 patients were treated by laparoscopic method and 52 patients were treated by open herniotomy method. The outcome of two techniques compare were operative time, recovery and discharge within 24 hours, post-operative pain assessment, cosmesis and complications.

Results: The mean operating time for open herniotomy was significantly less (36.69) as compared to that for laparoscopic hernia group (66.98). Recovery and discharge within 24 hours of patients undergoing laparoscopic hernia repair were significantly more $(78.85 \%)$ than the open herniotomy group (57.69\%). The CHIPPS for post-operative pain assessment were 5.28 for open herniotomy Vs 5.07 for laparoscopic hernia repair. FLACC tool for postoperative and periprocedural pain assessment was 4.75 for open hernioromy Vs 4.61 for laparoscopic hernia repair. The excellent cosmesis after laparoscopic procedure, whereas good cosmesis after open procedure among all subjects from respective groups. The complications including scrotal edema, erythema, hydrocele and recurrence were not significantly

Dr. Yuvraj Pawaskar

Department of General surgery, Government Medical College and Hospital Nagpur, Maharashtra, India-440003

How to cite this article:

Arti Mitra, Unmed Chandak, Yuvraj Pawaskar, Shiv Kumar Sahu, Sanskriti Sinha, Prasad Bansod, Nilesh Nagdeve, Akanksha Waldia. A comparative study between laparoscopic hernia repair and open herniotomy of inguinal hernia in paediatric age group: A prospective randomized controlled study. American Journal of Surgical Research and Reviews, 2021; 4:24.

\section{eScîPub}

eSciPub LLC, Houston, TX USA. Website: https://escipub.com/

By using the site/services, you are agreeing to our Policies: https:// escipub.com/terms-privacy-policydisclaimer/ 
different in the two groups. Conclusion: The study concludes that the well performed conventional herniotomy yields results similar to those of laparoscopic repair.

Keywords: Inguinal hernia, Laparoscopic hernia repair, Open herniotomy, Paediatric, Cosmesis, Recovery

\section{Introduction}

Pediatric inguinal hernia $(\mathrm{PIH})$ is a common disease in children, with incidence rates ranging from 0.8 to $4.4 \%{ }^{[1]}$. The treatment for this condition is high ligation of patent processus vaginalis at the level of the internal inguinal ring. This can be achieved either by conventional open method or by laparoscopic technique. There have been various studies regarding the place of laparoscopy in the treatment of inguinal hernia in children. At tertiary centres of India, laparoscopic inguinal hernia repair is not only possible in children, but also is gaining ground as a safe, feasible, and popular method. There are, however, several reports comparing the conventional open method and laparoscopic inguinal hernia repair in children, but the current trend is toward laparoscopic method [2].

However, with increasing interest in laparoscopic inguinal hernia repair, several treatment techniques have developed over the past two decades, aimed at improving the outcome. The various techniques differ in their approach to the internal inguinal ring, suturing and knotting techniques, endoscopic instruments used, mode of dissection of the hernia sac, The emerging techniques show a tendency for simple extracorporeal suturing and knotting technique, and diminished use of endoscopic ports and instruments [3]. The aim of this study was to review the role of laparoscopy in inguinal hernia repair in children, the intracorporeal laparoscopic surgical techniques, their current trend in paediatric surgical practice and complications.

\section{Materials and Methods}

After obtaining Institutional Ethics Committee (IEC) approval and written informed consent from parents/guardian, this prospective randomized comparative study was conducted in a Tertiary Care Centre of Central India over a period from May 2018 - November 2020. A total of 104 healthy children diagnosed with inguinal hernia, less than 12 years of age, who were operated with open and laparoscopic hernia repair, were sequentially enrolled in the study and were alternately randomized into two groups of 52 patients in each. Exclusion criteria were recurrent and complicated cases, hernia of canal of nuck in females, inguinal hernia with undescended tests and parental refusal.

Patients were admitted 1 day before surgery to hospital for routine preoperative evaluation and screened for associated problems like undescended testis. A detailed history, clinical signs and symptoms, the duration and blood investigations were carried out. Questionnaires were translated to local language i.e. Marathi and back translated to English for validation and then were used. Pre-operatively patients were kept nil per oral for 6 hours before surgery along with intra-venous fluids. Pre-anaesthetic check-up was done for all patients. At the time of induction of anaesthesia, antibiotic cefotaxime $50 \mathrm{mg} / \mathrm{kg}$ was administered. Parenteral analgesia (paraceta$\mathrm{mol} 15 \mathrm{mg} / \mathrm{kg} /$ dose every 6 hours) was given on the day of surgery and later switched to oral medication.

Alternate patients were subjected to laparoscopic group and open group. Open herniotomy was performed using a skin crease incision. Identification of the cord structures along with the sac done. High ligation of the sac was performed using 4-0/3-0 absorbable (Monocryl) suture. The wound was closed in layers, using absorbable suture.

Laparoscopic hernia repair was performed under general anaesthesia. The patient was positioned supine, often in the Trendelenberg position. Most descriptions position the surgeon on the side contralateral to the hernia with the monitor 
on the ipsilateral side. The abdomen is insufflated, typically through the umbilicus, to a pressure of $8-15 \mathrm{mmHg}$ depending on the size of the child. A variety of sizes of trocars, cameras, and instruments can be used, with the goal of smaller incisions. Any hernia contents were reduced before beginning repair of the hernia. Intracorporeal technique for laparoscopic hernia repair has been performed- all suturing and knot tying were done within the abdominal cavity with laparoscopic instruments. All laparoscopic procedures were completed without being converted to open procedure. Patients were operated by experienced, assistant professors and above.

Procedure indications, peculiarities, complications rates, recurrence rate and outcome were evaluated. Patients were followed up 6 hourly, 24 hourly, 2 days, 1 week, 1 month, and 6 months. Post-operative and periprocedural pain assessment was done by FLACC (face, legs, activity, cry, consolability) tool whereas CHIPPPS (children and infants post procedure pain score) for post-operative pain assessment.

\section{Statistical Analysis}

Data was entered in Microsoft excel sheet and analyzed using statistical software Epi Info. (7.2.1.0). Appropriate statistical test was applied. Descriptive statistics/data presented in tabular form with mean, standard deviation, range and wherever required tables, bars and graphs. Chi square test was applied for categorical data. $\mathrm{P}$ value $<0.05$ was considered significant.

\section{Observations and Results}

A total 104 healthy children of age $<12$ years diagnosed with inguinal hernias were enrolled and alternately randomized into two groups of 52 patients in each group. In both the groups, the maximum numbers of study subjects were in the age group of 1 to 5 years followed by 6 to 10 years with male predominance as shown in table 1 .

Table 1: Demographic data of the patients

\begin{tabular}{|c|c|c|c|c|}
\hline \multicolumn{2}{|c|}{ Demographic data } & Open herniotomy & Laparoscopic repair & P value \\
\hline \multirow{4}{*}{ Age } & $<$ 1year & $11(21.15 \%)$ & $00(0.0 \%)$ & \multirow{2}{*}{-} \\
\cline { 2 - 4 } & 1 to5 years & $20(38.46 \%)$ & $24(46.15 \%)$ & \\
\cline { 2 - 4 } & 6 to 10 years & $18(34.62 \%)$ & $24(46.15 \%)$ & \\
\cline { 2 - 4 } & 11 to 12 years & $03(5.77 \%)$ & $04(7.69 \%)$ & \multirow{2}{*}{0.274} \\
\hline \multirow{3}{*}{ Sex } & Male & $40(76.92 \%)$ & $35(67.31 \%)$ & - \\
\cline { 2 - 5 } & Female & $12(23.08 \%)$ & $17(32.69 \%)$ & \\
\cline { 2 - 4 } & Male: Female ratio & $3.33: 1$ & $2.05: 1$ & \\
\hline
\end{tabular}

The right side was commonly involved among both study groups (Figure 1). We observed bilateral inguinal hernias were commonly operated laparoscopically in 10 patients, of these $2(20 \%)$ were presented as bilateral and $8(80 \%)$ as unilateral hernias. Out of these 8 unilateral hernias, $6(75 \%)$ had contralateral patent processus vaginalis and intraoperative decision was taken to repair the contralateral side.

Figure 1: Comparison of sides' involvement between two groups

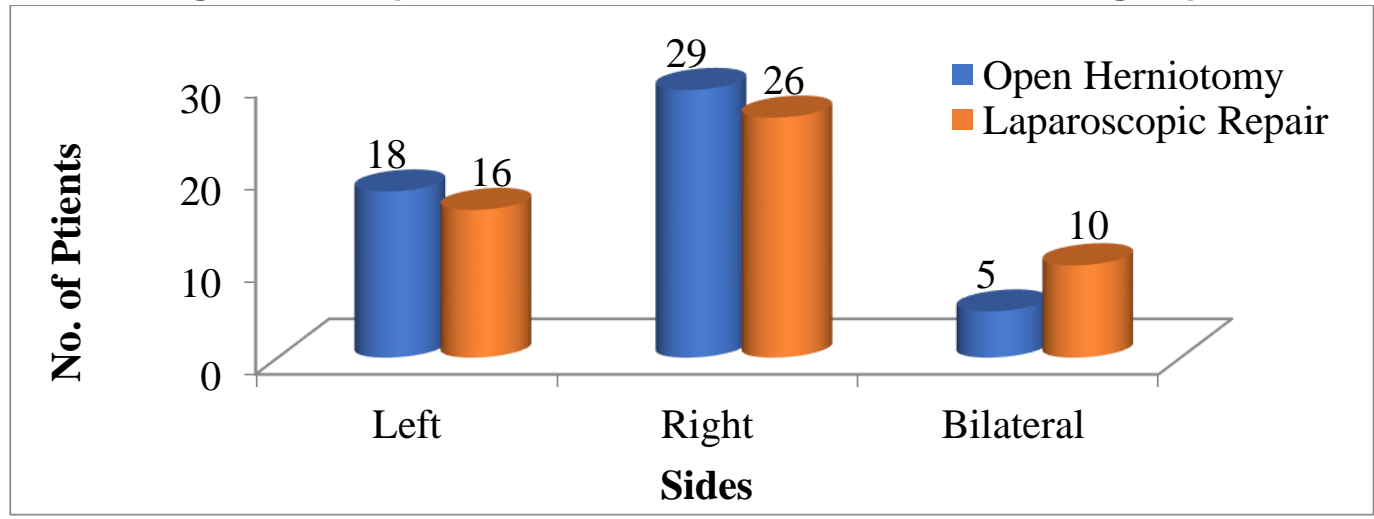


The operative details are shown in table 2. There was significant recovery within 24 hours in laparoscopic study group, $(41 ; 78.85 \%$ in laparoscopic vs. $30 ; 57.69 \%$ in open group, $p=0.0204$ ).
We found excellent cosmesis after laparoscopic procedure, whereas good cosmesis after open procedure among all subjects from respective groups.

Table 2: Operative time and post-operative pain assessment

\begin{tabular}{|c|c|c|c|}
\hline Operative details & Open herniotomy & Laparoscopic repair & P value \\
\hline Mean Operative time(minutes) & 36.69 & 66.98 & $<0.00001$ \\
\hline CHIPPS for post-operative pain assessment & 5.28 & 5.07 & 0.171 \\
\hline $\begin{array}{c}\text { FLACC tool for postoperative and periprocedural } \\
\text { pain assessment }\end{array}$ & 4.75 & 4.61 & 0.244 \\
\hline
\end{tabular}

The complications including scrotal edema, erythema, hydrocele and recurrence were not significantly different in the two groups as shown in table 3.

Table 3: Comparison of complications between two groups

\begin{tabular}{|c|c|c|c|c|}
\hline \multicolumn{2}{|c|}{ Complications } & $\begin{array}{c}\text { Open herni- } \\
\text { otomy }\end{array}$ & Laparoscopic repair & P value \\
\hline \multirow{2}{*}{ Early complications } & Scrotal edema & $9(17.31 \%)$ & $2(3.85 \%)$ & 0.0256 \\
\cline { 2 - 5 } & Erythema & $10(19.23 \%)$ & $0(0.0 \%)$ & - \\
\cline { 2 - 5 } & Hydrocele & $2(3.85 \%)$ & $4(7.69 \%)$ & 0.538 \\
\hline \multirow{2}{*}{ Late complications } & $\begin{array}{c}\text { Recurrence after } \\
6 \text { months }\end{array}$ & $2(3.85 \%)$ & $2(3.85 \%)$ & 0 \\
\hline
\end{tabular}

\section{Discussion}

Inguino-scrotal swellings in children form a majority of surgical conditions requiring treatment. Parents are usually the first person to notice the swelling or bulge. USG is a good alternative tool for diagnosing contralateral patent processus vaginalis. Traditional open hernia repair (OHR) has been implemented in the clinic for many years, which has the characteristics of simple operation and strong popularity ${ }^{[4]}$. Some scholars believe that it is necessary to open the inguinal canal, which causes postoperative pain, and it is easy to damage spermatic vessels and the vas deferens ${ }^{[5]}$. However, laparoscopic hernia repair (LHR) has the advantages of having a concealed scar, being minimally invasive, and having the ability to detect the contralateral patent processus vaginalis. Many authors think that LHR may gradually replace OHR and become the main surgical method for pediatric inguinal hernia ${ }^{[6,7]}$. Therefore, current prospective study was conducted to discuss effectiveness of two methods of pediatric inguinal hernia repair; laparoscopic with open methods.
The majority of study subjects in open herniotomy group were in age group of 1 to 5 years $(38.46 \%)$ while in laparoscopic hernia repair group majority of the study subjects belonged to the age group of 1 to 5 and 6 to 10 years (46.15\% each). These findings are correlated with the previous studies ${ }^{[8,9]}$. Regarding the sex prevalence, males are more commonly affected. The number of male patients was more as compared to the female patients in current study. This may be due the overall gender ratio in our country, or also due to negligence towards the female child. The childhood inguinal hernias are generally more predominant on the right side as found in present study and this has been attributed to the delay in descent of the right testis. The sex distribution and side involvement are comparable with the earlier studies [10-13].

An inguinal hernia will not resolve spontaneously and should be repaired as soon as possible after the diagnosis because of the risk of incarceration or strangulation. In general, infants and children require general anaesthesia for the operative repair of inguinal hernia. Recovery from the effects 
of anesthesia was delayed in a greater proportion of patients undergoing laparoscopic surgery. This may be due to deeper anesthesia and muscle relaxation needed for intubations in laparoscopic surgery ${ }^{[14]}$. Open surgery can well be performed with the patient under a face mask, especially when caudal analgesia is administered simultaneously. Even when a patient is intubated, the degree of anesthesia and relaxation needed is less. In contrast to this in present study there was significant recovery within $24 \mathrm{hrs}$ in laparoscopic study group which is in accordance with the study conducted by Saranga Bharathi et al [10].

Laparoscopic hernia repair in children is known to take longer operative time than open herniotomy. Many reports showed that it ranged from 50 to 94 minutes. However, the operative time is reduced with experience. It is well documented that the limiting step in laparoscopic hernia repair is the intracorporeal suturing of the internal inguinal ring. In open herniotomy, time is consumed in gaining access, obtaining adequate exposure, in localizing and isolating the sac from the cord structures. In laparoscopic surgery, approaching the hernial defect from within the abdomen, makes the area of interest bloodless, and the magnification renders anatomy very clear, making surgery precise. With growing experience and use of refinements, operative time does come down. In present study, the mean operative time for laparoscopic hernia repair was double the time for open herniotomy and the difference was statistically significant.

The CHIPPS for post-operative pain assessment were 5.28 for open herniotomy vs 5.07 for laparoscopic hernia repair. FLACC tool for postoperative and periprocedural pain assessment (4.75 for open hernioromy vs 4.61 for laparoscopic hernia repair). The result of CHIPPS and FLACC was not significant at $p<0.05$. These findings are correlated with the other studies ${ }^{[10,15]}$. Five-millimeter incisions in laparoscopic surgery were, indeed cosmetically more appealing compared with $2 \mathrm{~cm}$ incisions in open surgery. However, this significance gets lost because the scar in OS, by virtue of its position, gets concealed by clothing. We observed excellent cosmesis after laparoscopic procedure, whereas good cosmesis after open procedure among all subjects from respective groups which is comparable with the study carried out by Saranga Bharathi et al ${ }^{[10]}$. Post-operative complications like scrotal edema, erythema, hydrocele and recurrence are usually rare following elective operation. Recurrence is usually rare if operated by experienced surgeons but it can occur. A significant number of children presenting with unilateral hernias had contralateral patent processus vaginalis (75\%). The scrotal edema and Erythema more common among open procedure group (17.31\% each) while Hydrocele was more common among laparoscopic group. We observed similar recurrence among both study groups (3.85\% in each group). Reports from most studies $[10,15]$ document an incidence of $1 \%$ to $2 \%$. The recurrence may be associated with comorbid conditions including increased abdominal pressure, prematurity, malnutrition, and anemia and connective tissue disorders. However, it is unable to identify any clear benefit of laparoscopic inguinal herniotomy over open herniotomy apart from cosmesis and the ability to operate contralateral patent processus vaginalis.

\section{Conclusion}

The study concludes that the well performed conventional herniotomy yields results similar to those of laparoscopic repair. Cosmesis and the ability to detect and simultaneously repair CPPV are the 2 main advantages of laparoscopic hernia repair over open herniotomy. Keeping in view the low incidence of metachronicity in unilateral hernias, relative insignificance of cosmesis over the groin, and the constraints of the developing world, conventional open herniotomy can justly be performed for unilateral hernias, as the standard of care, in centers lacking facilities lacking laparoscopy. In developed countries laparoscopic paediatric inguinal hernia surgery is a novel, safe, elegant technique and in some situations advantageous too.

\section{Drawbacks:}


The medical facilities and monetary affluence are unevenly distributed in rural and urban areas in developing countries. Hence, the need is to find the apt treatment that is both scientifically/ethically correct while being cost effective. But the cost of setting up and running of laparoscopic set-up it may make it an unviable option in rural settings, where the majority of the developing world resides. Well-performed conventional herniotomy does yield equally good results and, therefore, needs to continue as the standard of care in centres where laparoscopic facilities are not available.

\section{References}

[1] Zhu LL, Xu WJ, Liu JB, Huang X, Lv ZB. Comparison of laparoscopic hernia repair and open herniotomy in children: a retrospective cohort study. Hernia. 2017;21(3):417-23.

[2] Scherer LR 3rd, Grosfeld JL. Inguinal hernia and umbilical anomalies. Pediatr Clin North Am. 1993;40(6):1121-31.

[3] Skinner MA, Grosfeld JL. Inguinal and umbilical hernia repair in infants and children. Surg Clin North Am. 1993;73(3):439-49.

[4] Zhou X, Qi X, Jiang B, Sha Y, Song D. Transumbilical endoscopic technique for complete closure of inguinal hernias in female pediatric patients. Exp Ther Med. 2017;13(1):41-4.

[5] Zhou J, Chen X, Jiang T. Pediatric inguinal hernia treated by single-port laparoscopic water injection hernia crochet needle. Wideochir Inne Tech Maloinwazyjne. 2020;15(1):239-44.

[6] Davies DA, Rideout DA, Clarke SA. The Interna-
[7] tional Pediatric Endosurgery Group EvidenceBased Guideline on Minimal Access Approaches to the Operative Management of Inguinal Hernia in Children. J Laparoendosc Adv Surg Tech A. 2020;30(2):221-7.

[8] Xiao Y. Single-port laparoscopic percutaneous extraperitoneal closure for inguinal hernias repair in girls: using an epidural needle assisted by a towel forceps. BMC Surg. 2020;20(1):139.

[9] Okunribido O. Ladipo J.K. and Ajao O.G. "Inguinal hernia in paediatric age group, Ibadan experience", East Afr. Med. J.,1992; 69 (6): 347-348.

[10] Adesunkanmi A.R., Adejuyigbe O: "Prognostic factors in childhood inguinal hernia at Wesley Guild Hospital,Nigeria", East. Afr.Med. J, 1990;76 (3) :144-147.

[11] Saranga Bharathi R, Arora M, Baskaran V. Pediatric inguinal hernia: laparoscopic versus open surgery. JSLS. 2008 Sep;12(3):277-81.

[12] Grosfeld J .L Weber T.R- "congenital abdominal wall defects" current problems in surgry 1982; 19;159.

[13]F Ralph M. Larsen and Nashville Tenn: "Inguinal Hernia in Infancy and Early childhood", Annals of Surgery; 1949;25:307-328.

[14] Gilbert Al: Inguinal hernia repair: biomaterials and sutureless repair. Perspective Gen Surg. 1991; 2:113-129.

[15] Wills VL, Hunt DR. Pain after laparoscopic cholecystectomy. Br J Surg. 2000;87:273-284.

[16] Elekiabi, Omar \& Eraky, Mohamed \& Abdelhady, Waleed \& Sallam, Ahmed \& Gertallah, Loay. A Comparative Study between Laparoscopic Repair and Open Repair of Pediatric Inguinal Hernia; Outcomes and Benefits. Acta Scientific Paediatrics 2019; 2:03-08. 